\title{
Influence of Stationary and Time-Varying Periodical Interference on Magnetic Sensor Noise Analysis Using Allan Variance
}

\author{
K. Draganová ${ }^{a, *}$, M. LašŠÁK ${ }^{b}$, P. LipovskÝ ${ }^{a}$, M. Šmelko ${ }^{a}$, J. BlažeK ${ }^{a}$, K. SemráD ${ }^{a}$, \\ A. TOBISOVÁ ${ }^{a}$ AND M. KOŠUdA ${ }^{a}$ \\ ${ }^{a}$ Technical University of Kosice, Faculty of Aeronautics, Rampová 7, 04121 Košice, Slovak Republic \\ ${ }^{b}$ Flight Management and Control, CoE, Honeywell International, Inc., \\ Tuřanka 100, 62700 Brno, Czech Republic
}

\begin{abstract}
The Allan variance methodology is a statistic approach, which can be used for the analysis of the noise processes. It can be used for the noise type determination of inertial sensors without the need of any transformations. Although as the Allan variance is a time domain measure, the article deals with the methodology of how to reveal the periodical interference during the noise analysis of magnetic sensors. Subsequently the methodology based on the dynamic Allan variance is applied for the cases, when the periodical interference is non-stationary and varies in time. The noise analysis determining the noise specific types is inter alia very beneficial for the in-site or in-flight magnetometer calibration.
\end{abstract}

DOI: 10.12693/APhysPolA.137.677

PACS/topics: Allan variance, magnetic sensor, periodical interference

\section{Introduction}

Noise analysis of magnetic sensors can be performed using many methodologies. One of the advantageous methods supported also by the IEEE is the noise analysis using the Allan variance. This method is based on the analysis of a data sequence in the time domain and can also be used to determine the noise types as a function of the averaging period. Currently many optimization methods have been researched [1-3]. We proved that the theory, with some modifications and simplifications, can be applied also for determining the noise type of magnetic sensors [4]. These sensors are nowadays, together with accelerometers and gyroscopes, a common part mainly of the small inertial measurement units. Furthermore, during the noise analysis, it is necessary to consider the characteristics resulting from the time-varying noise amplitude [5]. The Allan variance analysis is the most common time domain measure. However, for the application of the noise analysis using the Allan variance it is advantageous to have methodology to reveal the periodical interference during the noise analysis even in the cases where the periodical interference is non-stationary. This is very important especially if the in-site or in-flight calibration with no magnetic chamber is performed. It is also very advantageous for the utilization of the calibration methods based on the neural networks, which can be influenced by the particular noise types [6].

*corresponding author; e-mail: katarina.draganova@tuke.sk

\section{Stationary periodical interference}

The methodology for the noise analysis using the Allan variance that we applied to the magnetic sensors is in detail described in [4]. However, the methodology does not involve the influence of the periodical interference on the noise analysis. That was the reason why the simulation model representing output sensor signal with the duration of $1000 \mathrm{~s}$ using the sampling frequency of $1 \mathrm{kHz}$ was created.

In the first simulation the signal involved also the $50 \mathrm{~Hz}$ industrial frequency and three output signals marked as $y_{0}, y_{1}$, and $y_{2}$ were used. The output signal varied only in the noise amplitude of the signal, which was set to 1 in the case of the $y_{0}$ signal. The amplitude of the periodical interference was ten times higher for the signal $y_{1}$ and ten times lower for the signal $y_{2}$.

The results of the noise analysis using the Allan variance methodology with non-overlapping samples were processed and visualized in the form of the log-log plots. One of the advantage of this methodology is that the noise type can be clearly seen because it is dependent on the slopes of the curves in the particular regions of the averaging period $\tau$. From the results shown in Fig. 1 significant deviations from the ideal linear slopes can be seen in the form of the peaks caused by the periodical interference. Furthermore, it can be seen that the periodical interference has no influence on the noise type. The calculated characteristics vary only in the amplitudes. Although in comparison to the sensor output signals that varied with the step of one decade, it is necessary to take into account that in case of the Allan variance analysis this difference is two decades. 


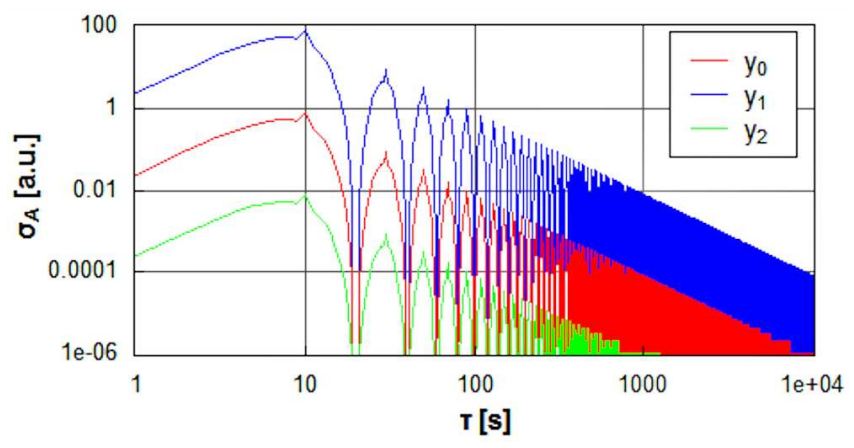

Fig. 1. Allan variance calculated for the stationary periodical interference signals with different amplitudes.

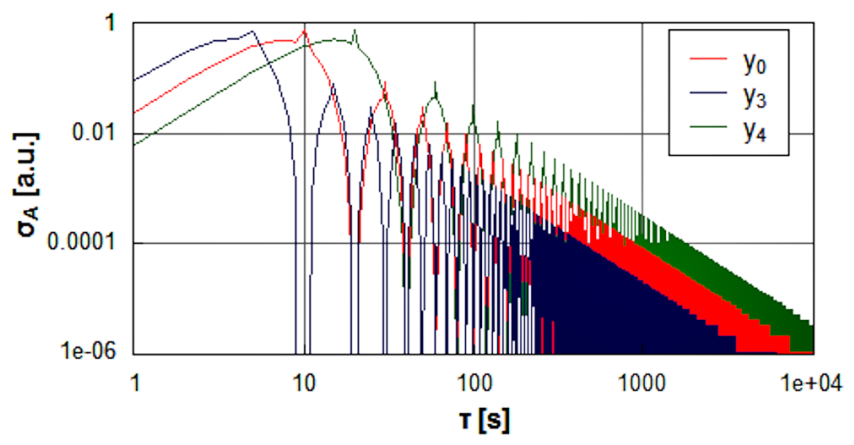

Fig. 2. Allan variance calculated for the stationary periodical interference signals with different frequencies.

The second series of simulations were focused on the influence of the changing frequency of the interference signal. In this case the signal $y_{0}$ was compared to other two signals varying only in the used frequency of the interference signal. In case of the $y_{3}$ signal it was $100 \mathrm{~Hz}$ and in case of the $y_{4}$ signal it was $25 \mathrm{~Hz}$. The influence of the frequency of the periodical interference can be clearly seen from the simulation results shown in Fig. 2. If the frequency of the interference is twice higher or lower, we get in the Allan variance characteristics twice more or less number of peaks, respectively. It is important to notice that the amplitudes of the peaks remain the same, which is very important for the determination of the noise type parameters.

Consequently, the simulation results were verified experimentally. Measurements were performed using the VEMA-04 magnetometer based on the magnetic microwires [7]. In Fig. 3 two data sets are shown. The sensor signal marked as $y_{m 1}$ represents the reference signal with the unfiltered industrial $50 \mathrm{~Hz}$ frequency and the sensor signal designated as $y_{m 2}$ represents the output signal of the sensor, in which the transformer load was switched on near the sensor, which caused a significant difference in the interference signal amplitude, whereas the dominating frequency remained the same. The standard deviation of the measured signal changed from $52 \mathrm{nT}$ to $488 \mathrm{nT}$.

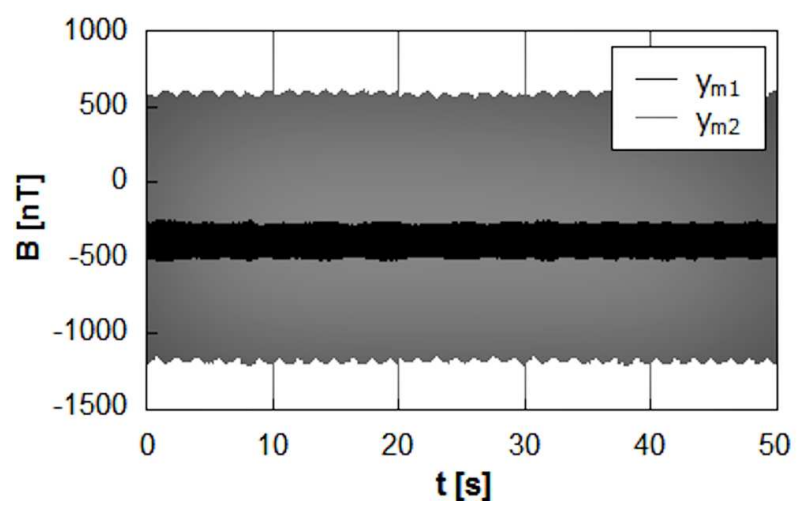

Fig. 3. Measured signal with the stationary periodical interference.

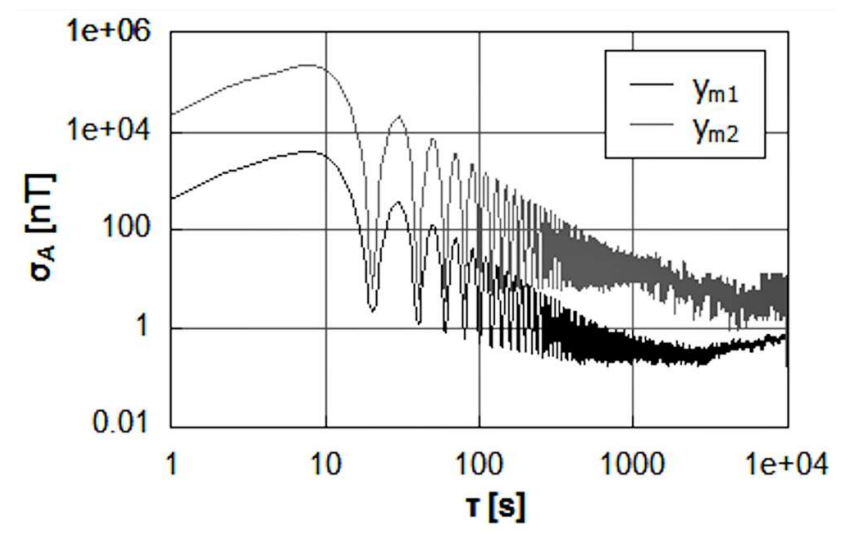

Fig. 4. Allan variance calculated for the measured stationary periodical interference.

In Fig. 4 the Allan variance calculated for the $y_{m 1}$ and $y_{m 2}$ signals is shown. From the calculated characteristics we can see that for lower averaging period until the $10^{3} \mathrm{~s}$ the white or quantization noise is dominant. Then the slope changes to the random walk due to the bias instability in the sensor output in case of the $y_{m 1}$ signal. A very similar trend is observed also in the case of the $y_{m 2}$ signal with negligible differences of the slopes mainly in the higher averaging periods, where the influence of the bias instability occurs. However, the significant difference can be seen similarly to the performed simulations in the amplitudes. Oscillations in the characteristics are caused by the dominant $50 \mathrm{~Hz}$ industrial frequency.

\section{Time-varying periodical interference}

In addition to the changing amplitude or frequency of the examined signal with the periodical interference, the non-stationary behavior over time was simulated. In the first case of the simulated signal $y_{5}$, the step change of the periodical interference amplitude between the $y_{0}$ and $y_{1}$ in the time of $0.5 \mathrm{~s}$ was analyzed. Analogically, in case of the $y_{6}$ signal, the step change of the periodical interference amplitude between the $y_{1}$ and $y_{0}$ signals in the 


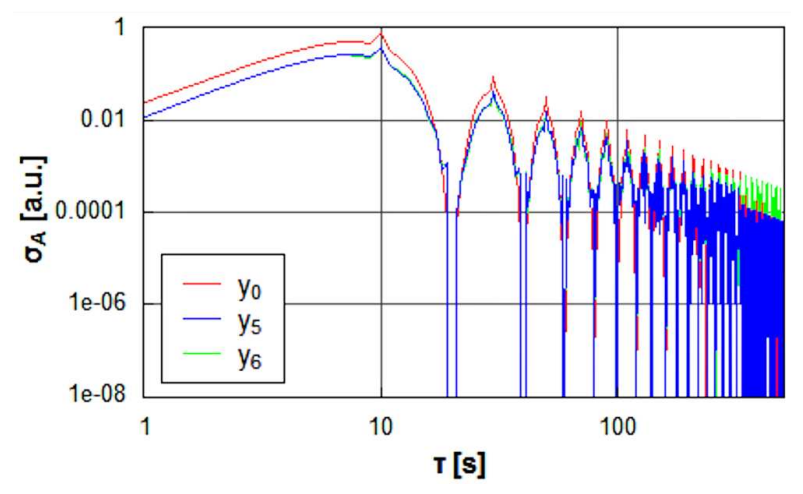

Fig. 5. Allan variance calculated for non-stationary periodical interference signals with different amplitudes.

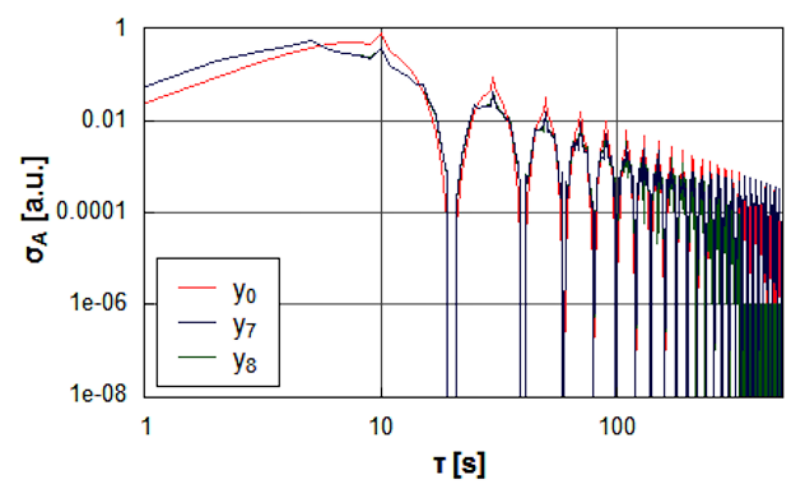

Fig. 6. As in Fig. 5, but for different frequencies.

time of $0.5 \mathrm{~s}$ was analyzed. The second simulation model was created so that the signal marked as $y_{7}$ involved step change of the frequency in the time of $0.5 \mathrm{~s}$ between the $y_{0}$ and $y_{3}$ signal (from $50 \mathrm{~Hz}$ to $100 \mathrm{~Hz}$ ) and analogically in the signal $y_{8}$, the step change of the frequency from $y_{3}$ to $y_{0}$ was simulated. Figures 5 and 6 show the comparison of the simulation results and the reference $y_{0}$ signal. For the purposes of the time-varying periodical interference signal analysis the simulation model consisting only $10^{3}$ samples was used.

The reason can be clearly seen from Fig. 5 and Fig. 6 . The deviations among the signals can be seen only for the higher values of the averaging period and from the models created from more samples as they are negligible in the $\log -\log$ scale. The simulation results also confirmed that in case of the non-stationary noise analysis of the signals involving the non-stationary periodical interference, the conventional Allan variance approach is not satisfactory for the noise analysis. That is the reason why we decided to apply the theory of dynamic Allan variance for the noise analysis of the magnetic sensor signals with the time-varying periodical interference in more details described in [8]. The theory was confirmed by experimental measurements performed using the same measurement chain as in case of the stationary measurements. The non-stationary sensor signal $y_{m 3}$ involved

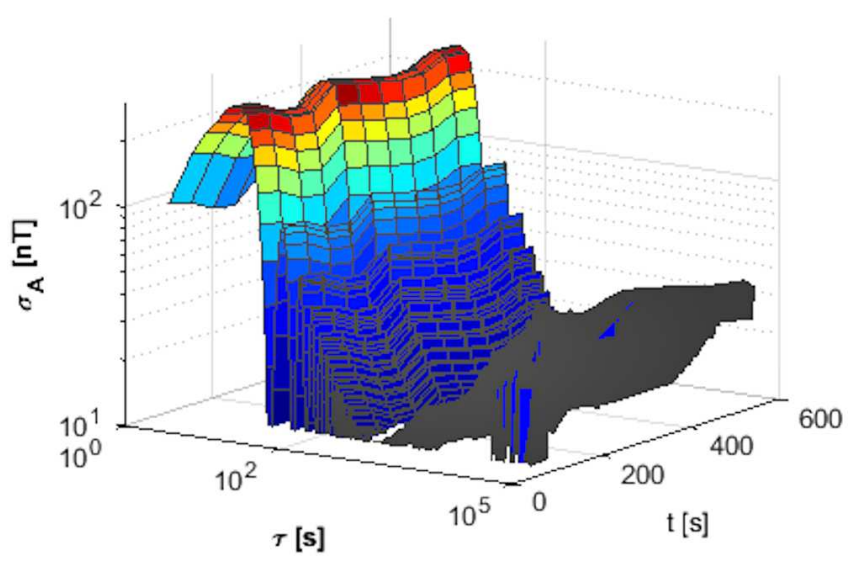

Fig. 7. Allan variance calculated for the measured non-stationary periodical interference.

the step changes of the interference signal amplitudes, in which the transformer load was switched on near the sensor in three time intervals - after 60, 360, and $510 \mathrm{~s}$ with the duration of $60 \mathrm{~s}$.

From the calculated characteristics shown in Fig. 7, we can see that for lower averaging time until $10^{3} \mathrm{~s}$ the white or quantisation noise is dominant. Then the slope changes to the random walk due to the bias instability in the sensor output. The non-stationary periodical interference caused only negligible differences of the slopes, but the significant influence can be seen in the form of the oscillations in the characteristics and also in the amplitudes in the time intervals, when the transformer was switched on.

\section{Conclusions}

The statistical methodology of the noise analysis based on the Allan variance in comparison to other conventional statistical methods has several significant advantages. There is no need of any transformation, which is one of the preferred methods for the identification of the inertial sensors noise types. As it was confirmed in the article, in the conventional form or in the extended version named dynamic Allan variance, it can be very effectively used for the revelation of the periodical interference in the inertial sensor output signal. This was proved by the simulations and also by the experimental results for the stationary and non-stationary interference behavior, which is very important for the selection of the suitable calibration algorithm especially if the in-site calibration based on the neural networks is applied.

\section{Acknowledgments}

This work was supported by the APVV-17-0184, APVV-18-0248, VEGA No. 1/0374/17, KEGA No. 052TUKE-4/2018, and KEGA No. 058TUKE-4/2018 projects. 


\section{References}

[1] S.Y. Xu, F.F. Xing, W. Li, Y.Q. Wang, J. Central South Univ. 24, 11 (2017).

[2] M. Liu, L. Gui, M. Huang, J. Computat. Theor. Nanosci. 1, 8 (2016).

[3] M. Matejcek, M. Sostronek, in: Proc. 9th Int. Sci. Conf. on Communication and Information Technologies, KIT, Vysoke Tatry (Slovakia), 2017.

[4] K. Draganová, F. Kmec, J. Blažek, D. Praslička, J. Hudák, M. Laššák, Acta Phys. Pol. A 126, 394 (2014).
[5] R. Song, X. Chen, H. Huang, Sens. Actuat. A 276 , (2018).

[6] T. Kliment, P. Lipovský, K. Draganová, V. Moucha, in: Proc. Int. Conf. on New Trends in Signal Processing (NTSP), Demanovska Dolina (Slovakia), 2018.

[7] K. Richter, R. Varga, A. Thiaville, IEEE Trans. Magn. 50, (2014).

[8] K. Draganová, V. Moucha, T. Volcko, K. Semrád, Acta Phys. Pol. A 131, 4 (2017). 